\title{
Long-Term Semantic Memory Versus Contextual Memory in Unconscious Number Processing
}

\author{
Anthony G. Greenwald and Richard L. Abrams \\ University of Washington
}

\author{
Lionel Naccache \\ Service Hospitalier Frederic Joliot; \\ and Hôpital de la Pitié-Salpêtrière
}

\author{
Stanislas Dehaene \\ Service Hospitalier Frederic Joliot
}

\begin{abstract}
Subjects classified visible 2-digit numbers as larger or smaller than 55. Target numbers were preceded by masked 2-digit primes that were either congruent (same relation to 55) or incongruent. Experiments 1 and 2 showed prime congruency effects for stimuli never included in the set of classified visible targets, indicating subliminal priming based on long-term semantic memory. Experiments 2 and 3 went further to demonstrate paradoxical unconscious priming effects resulting from task context. For example, after repeated practice classifying 73 as larger than 55 , the novel masked prime 37 paradoxically facilitated the "larger" response. In these experiments task context could induce subjects to unconsciously process only the leftmost masked prime digit, only the rightmost digit, or both independently. Across 3 experiments, subliminal priming was governed by both task context and long-term semantic memory.
\end{abstract}

This research started by asking how much semantic analysis occurs unconsciously in response to visually masked numbers. Experiment 1 set out specifically to resolve a discrepancy between two recently reported findings. When it became apparent that Experiment 1's methods could address additional interesting questions about subliminal priming, those additional questions became the focus of Experiments 2 and 3.

The empirical discrepancy that motivated this research came from two subliminal priming experiments. Both experiments used two-choice categorization tasks, and both used visible target stimuli following masked primes that were either in the same category as targets (congruent) or not (incongruent). In these experiments, priming effects took the form of faster or more accurate performance (or both) on congruent than incongruent trials.

In the first of the problematic pair of studies, subjects' twochoice task was valence classification-classifying words as

Anthony G. Greenwald and Richard L. Abrams, Department of Psychology, University of Washington; Lionel Naccache, Institut National de la Santé et de la Recherche Médicale (INSERM) Unit 334, Service Hospitalier Frederic Joliot, Commissariat à l' Energie Atomique/Direction des Sciences de la Vie/Département de la Recherche Médicale (CEA/DRM/ DSV), Orsay, France and Clinical Neurophysiology Department, Hôpital de la Pitié-Salpêtrière, Paris, France; Stanislas Dehaene, INSERM Unit 334, Service Hospitalier Frederic Joliot, CEA/DRM/DSV.

This research was supported by National Institute of Mental Health Grants MH-41328, MH-01533, and MH-57672 to Anthony G. Greenwald. Stanislas Dehaene received support from INSERM and from a McDonnell Foundation Centennial Fellowship.

Correspondence concerning this article should be addressed to Anthony G. Greenwald, Department of Psychology, University of Washington, Box 351525, Seattle, Washington 98195-1525. E-mail: agg@u.washington.edu pleasant or unpleasant in meaning. In this study, Abrams and Greenwald (2000) found priming by masked words that were selected from the same set that subjects had been practicing classifying in visible form. However, priming did not occur for words that had never been presented visibly during the experiment Conceptually similar results were reported subsequently by Damian (2001). By contrast, in a study in which either Arabic numerals (e.g., 6) or words (e.g., six) were to be classified as less than or greater than five, Naccache and Dehaene (2001) obtained priming by masked stimuli that had never been presented visibly in the experiment.

Abrams and Greenwald's (2000) subjects practiced valence classification with a set of four-, five-, and six-letter words, after which they were tested with visually masked primes selected either from this same set of words (old set) or from another set (new set). Subliminal priming occurred only for the old set primes. Naccache and Dehaene's (2001) subjects practiced quantity classification (smaller or larger than five) with just four target numbers: 1, 4, 6, and 9 (old set). Visually masked primes included both these old set numbers and also four unpracticed numbers, 2, 3, 7, and 8 (new set). With this procedure, both the old and new sets functioned appropriately as visually masked primes, meaning that they produced faster responses to visible targets that had the same ordinal relation to 5 .

\section{Strategic Influences on Unconscious Cognition?}

At the rebirth of modern research interest in cognitive effects of subliminal visual stimuli, unconsciously perceived primes were assumed to be free of top-down, strategic effects (Marcel, 1983). Debate focused on methodological concerns (Holender, 1986). The main goal of researchers was to establish the existence of 
reproducible subliminal priming effects. More recently, evidence for reproducible subliminal priming effects has accumulated (see Draine \& Greenwald, 1998). Consequently, debate has moved beyond existence claims. An active focus of current debate is the question to which the present research was initially addressed: the extent of analysis received by visually masked stimuli. Specifically, for masked primes, does semantic analysis occur (Dell'Acqua \& Grainger, 1999; Naccache \& Dehaene, 2001) or does it not (Abrams \& Greenwald, 2000; Damian, 2001; Klinger, Burton, \& Pitts, 2000)?

Recent research on priming by visible (i.e., nonmasked) primes has yielded growing evidence for top-down influences on priming effects (see review by Smith, Besner, \& Miyoshi, 1994). A natural further development for the masked priming domain has therefore been to raise the question addressed by the present Experiments 2 and 3: What are the nature of top-down, or strategic, effects in masked priming (see Abrams \& Greenwald, 2000; Bodner \& Masson, 2001; Lachter et al., 2000; Naccache, Blandin, \& Dehaene, 2002)?

\section{Long-Term Semantic Memory Versus Contextual Memory}

One of Abrams and Greenwald's (2000) findings revealed a distinction that will be useful in describing the present Experiments 2 and 3. After subjects had practiced classifying visible, evaluatively negative words (e.g., bile and smut), the nonword biut - which is a hybrid in the sense that it is composed of parts of two practiced parent words-functioned as an evaluatively negative prime. This priming by hybrids was found even when the word parts combined to produce a word with opposite valence. For example, the hybrid smile (also created from bile and smut) functioned paradoxically as an evaluatively negative subliminal prime, despite its being a word with a consensually known, unambiguous, evaluatively positive meaning.

Abrams and Greenwald's (2000) strategy was to put priming effects based on consensual (i.e., dictionary) word meanings into opposition with ones based on associations that could only have been acquired during the experiment. Remarkably, effects of the within-experiment associations proved to be stronger. These two categories of associations (within-experiment and previously existing) do not map well onto existing memory dichotomies such as long-term versus short-term, semantic versus episodic, or stateindependent versus state-dependent memories. In the following, they will be referred to as long-term semantic memory versus contextual memory.

\section{Experiment 1}

Abrams and Greenwald (2000) found that masked words did not activate long-term semantic memories, but only contextual (within-experiment) associations. Contrariwise, Naccache and Dehaene (2001) found that long-term semantic meaning of numbers was activated by visually masked numbers. Experiment 1 was designed to determine whether this apparent contradiction could be resolved by replicating Naccache and Dehaene's experiment with the methods used by Abrams and Greenwald.

Three procedural differences might explain why Naccache and Dehaene (2001) found evidence for unconscious analysis of new-set number stimuli, whereas Abrams and Greenwald (2000) did not. First, and most obvious, the stimuli differed: the former study used numbers, whereas the latter study used words. Second, the measures of subliminal priming differed Naccache and Dehaene used a priming measure based on latency differences between congruent and incongruent priming trials, whereas Abrams and Greenwald's measure was based on accuracy differences. Abrams and Greenwald used the response window procedure, which forces very rapid responding to all target stimuli and may therefore bypass semantic analyses that take time to develop (see Draine \& Greenwald, 1998; Greenwald, Draine, \& Abrams, 1996). Third, masked primes in the two studies may have differed in visibility. Both experiments included, as tests of prime visibility, blocks of trials in which subjects classified masked primes into the same categories that were used for classifying visible target stimuli (i.e., positivenegative valence for Abrams \& Greenwald and ordinal relation to 5 for Naccache \& Dehaene). Mean performance on these visibility tests, assessed using the signal-detection sensitivity measure $\left(d^{\prime}\right)$, was greater for Naccache and Dehaene's procedure (mean $d^{\prime}=.6$ in their first experiment) than for Abrams and Greenwald's procedure (mean $d^{\prime}=.1$ ).

Experiment 1 was designed as a conceptual replication of Naccache and Dehaene's (2001) number classification experiment using the response window procedure. A modification of Naccache and Dehaene's task made possible a substantial increase in the number of stimuli that could be used. The modification was to ask subjects to judge quantity of two-digit numbers in relation to 55 .

The procedures of this and the following experiments were quite similar. All measured the influence of visually masked two-digit primes on the subsequent classification of two-digit target numbers. The differences among the experiments were limited to selections of two-digit numbers used as visible targets and as visually masked primes. In Experiment 1, numbers that were presented as primes were never presented as targets and were composed of digits that had never appeared in visible target numbers. The predictions are straightforward. If, as originally observed by Abrams and Greenwald (2000), masked stimuli do not activate long-term semantic memories, then no priming effect should be observed. However if, as proposed by Dehaene et al. (1998), masked numerals do access long-term semantic representations of numeric quantity, then the quantity represented by the prime (smaller or larger than 55) should influence the response to the quantity represented by the target.

\section{Method}

\section{Subjects}

Twenty-seven University of Washington undergraduates participated in exchange for credit toward a course requirement. All had normal or corrected-to-normal vision, were fluent in English, and were naive regarding the purposes of the experiment.

\section{Apparatus and Stimuli}

A desktop computer, a table, an adjustable-height chair, and a white noise generator were the furnishings of small laboratory rooms in which subjects completed the experiment. Subjects viewed the computer display (120 Hz refresh rate) from a distance of about $65 \mathrm{~cm}$. All stimuli were 


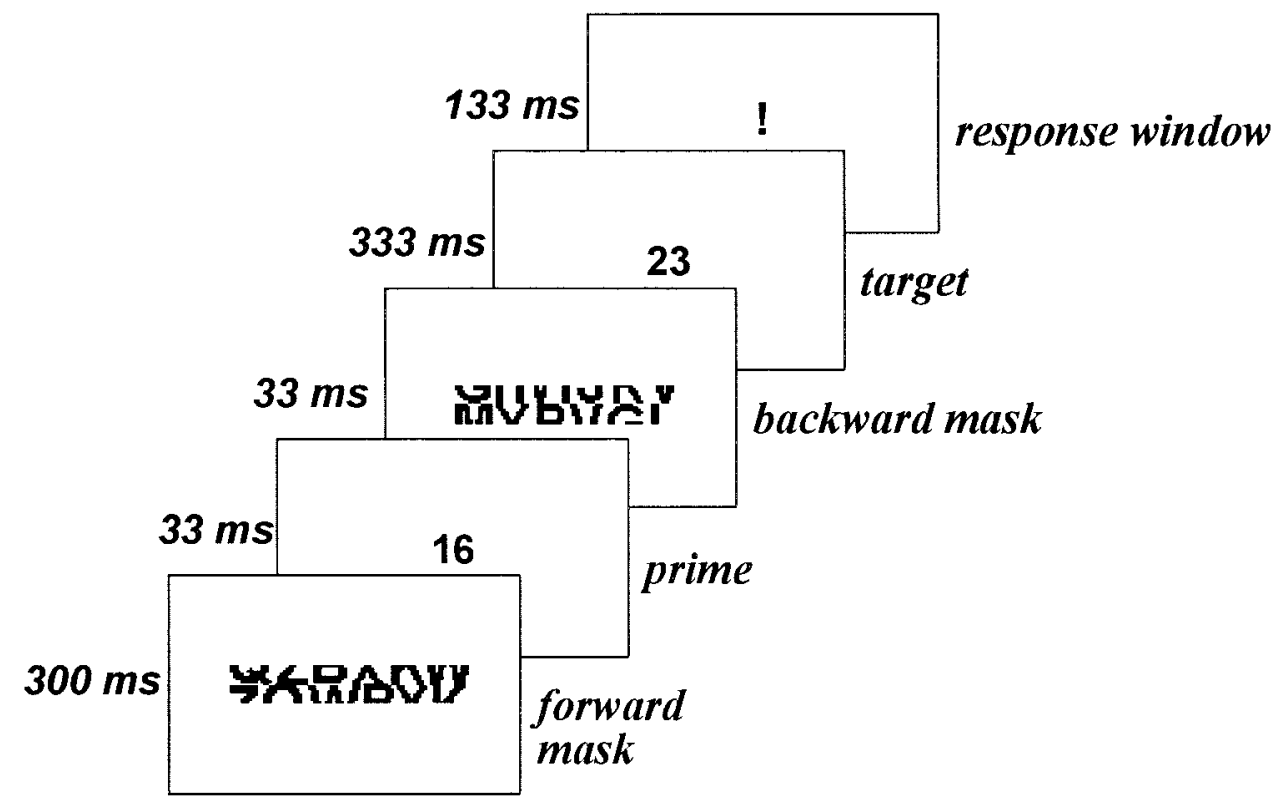

Figure 1. Sequence and durations of events on priming trials using the response-window procedure. This procedure was used for the priming task in all three of the present experiments.

presented centered in the computer display (43-cm diagonal screen size) as black digits in a white background rectangle, $5.3 \mathrm{~cm}$ high $\times 12 \mathrm{~cm}$ wide. ${ }^{1}$ The remainder of the screen was a medium gray. The two-digit prime and target stimuli were presented in Arial font, $1.0 \mathrm{~cm}$ high $\times 1.3 \mathrm{~cm}$ wide. Forward and backward masks, which were displayed just before and just after prime stimuli, consisted of letter fragments occupying a rectangular area $1.4 \mathrm{~cm}$ high $\times 7.0 \mathrm{~cm}$ wide. These masks were selected randomly on each trial from a set of eight such masks, with the constraint that the forward and backward masks would differ. The letter string "XXXX," 1.0 $\mathrm{cm}$ high $\times 2.5 \mathrm{~cm}$ wide, was used on practice trials in place of the prime stimulus.

\section{Stimulus Design}

Subjects performed at the quantity classification task with one set of visible two-digit target stimuli (old set), and were then tested for unconscious analysis by adding two-digit masked primes to the stimulus sequence on each trial (see Figure 1). The test procedure's visible stimuli (old set) and masked primes (new set) were mutually exclusive sets of two-digit numbers. (See Table 1 for all stimuli.) In the first of two counterbalancing sets of stimuli, visible target stimuli were the 24 two-digit numbers that could be constructed using only the digits $1,4,5,6$, and 9 (not including 55 ), whereas masked primes were the sixteen two-digit numbers that could be constructed using only the digits $2,3,7$, and 8 . The roles of the subsets $(1,4,6,9$ and $2,3,7,8)$ were interchanged for the second counterbalancing set.

\section{Indirect Measure-Test of Priming}

Practice. The sequence of stimuli in the first of four blocks of 48 practice trials consisted of (a) a plus sign in the center of the screen for 300 ms, (b) a 300-ms forward mask, (c) the letter string "XXXX" for $33 \mathrm{~ms}$ (this was the temporal position in which two-digit primes would appear after practice), (d) a 33-ms backward mask, and (e) a two-digit target stimulus that remained on screen until the subject responded to it.

In the first block of practice trials, subjects were asked to classify two-digit targets as less than 55, "Press the "D" key with the left index finger"; or greater than 55, "Press the "K" key with the right index finger." Instructions advised ignoring any stimuli that preceded the two-digit number, and explained that the word "ERROR" would appear immediately after any incorrect response. This error feedback appeared in the middle of the screen for $200 \mathrm{~ms}$. The interval between either (a) pressing the key for the correct response or (b) the end of the error feedback and the start of the next trial was $500 \mathrm{~ms}$. End-of-block feedback reported to the subject both mean latency and percent correct responses for the just completed block.

Response window. All tests of priming used a response window procedure (Draine \& Greenwald, 1998; Greenwald et al., 1996) that instructs subjects to give rapid responses and obliges them to maintain low variability of latency across trials. The speed pressure of the response window procedure necessarily produces a relatively high error rate. Priming effects can then be observed as substantially higher error rates for incongruent than for congruent priming trials.

The response window was introduced on the second block of practice. For the response window procedure, the two-digit target stimulus lasted $333 \mathrm{~ms}$ and was followed immediately by an exclamation mark, which stayed on screen for $133 \mathrm{~ms}$ and defined the response window interval. (See Figure 1.) Subjects were instructed to respond while the exclamation point was on the screen. To give subjects feedback that they had successfully responded during the window interval, the exclamation mark immediately turned red and persisted for $300 \mathrm{~ms}$. If the exclamation mark disappeared without turning red, subjects knew that they were too slow; and, if the exclamation mark did not appear at all, subjects knew they were too fast. As in the first (no-window) practice block, error feedback was given immediately after any incorrect response. A report of percent of responses successfully made during the window interval was added to the end-of-block summary report of mean latency and percent correct.

When the response window was introduced in the second practice block, the 133-ms window followed a 333-ms target stimulus, such that the

\footnotetext{
${ }^{1}$ Procedures were programmed using Inquisit (Millisecond Software, 1998), which runs on desktop computers with a Windows operating system.
} 
Table 1

Stimulus Design for Experiment 1

\begin{tabular}{lcc}
\hline $\begin{array}{c}\text { Counterbalancing set } \\
\text { and stimulus }\end{array}$ & Numbers less than 55 & Numbers greater than 55 \\
\hline $\begin{array}{l}\text { Counterbalancing Set A } \\
\text { Targets (old set) }\end{array}$ & $11,14,15,16,19,41$, & \\
& $44,45,46,49,51,54$ & $56,59,61,64,65,66$, \\
Masked primes (new set) & $22,23,27,28,32,33,37,38$ & $69,91,94,95,96,99$ \\
Counterbalancing Set B & $22,23,25,27,28,32$, & $72,77,78,82,83,87,88$ \\
Targets (old set) & $33,35,37,38,52,53$ & $78,88,72,73,75,77$, \\
& $11,14,16,19,41,44,46,49$ & $61,64,66,69,91,94,96,99$ \\
Masked primes (new set) & & \\
\hline
\end{tabular}

Note. Half of the subjects received the stimuli in Counterbalancing Set A, and half received the stimuli in Counterbalancing Set B. The two counterbalancing sets were formed by interchanging the roles of the digit subsets $(1,4,6,9)$ and $(2,3,7,8)$.

temporal center of the 133-ms response window interval occurred at 400 ms following target onset. After each block, the program controlling the experiment optionally advanced or delayed the window stimulus (exclamation point) by $33 \mathrm{~ms}$, for the purpose of maintaining an error rate of approximately $35 \% .^{2}$

Priming test. At the beginning of the fifth block, subjects were informed that data collection was starting and that error feedback would no longer be provided. Subjects were not alerted to the one critical change in procedure for the data collection blocks. In the prime's position in the stimulus sequence-a position occupied by the letter string "XXXX" during the first four blocks-two-digit masked primes were introduced according to the design shown in Table 1. With 48 trials in each block, each of the 24 target stimuli appeared twice and each of the 16 masked primes (see Table 1) appeared three times. These appearances were randomized, with the constraint that there were 24 congruent and 24 incongruent priming trials in each block. Congruent priming trials were ones for which prime and target both had the same ordinal relation to 55 . In all other respects, the five data collection blocks resembled the three preceding response window practice blocks. Subjects continued to receive feedback at the end of each block, reporting mean latency, percent correct, and percent of responses successfully made during the response window interval.

\section{Direct Measures-Tests of Prime Visibility}

On the 10th block, a new task was introduced, with the following instructions.

In the following blocks, you will ... be categorizing numbers that appear flashed very rapidly in the trial sequence just before the number you had been categorizing. . . . In this practice block, these numbers will be presented for a longer duration.... From now on, your responses do not need to be made while the exclamation mark is on the screen. Wait a bit longer before responding.

The test of prime visibility started with two practice blocks of 48 trials, in the first of which the prime duration was increased from $33 \mathrm{~ms}$ to 100 $\mathrm{ms}$, a duration that was sufficient to make the prime visible to all subjects. In the second practice block, prime duration was reduced to $67 \mathrm{~ms}$, which still allowed masked primes to be visible to most subjects. For both of these practice blocks subjects received error feedback immediately after any incorrect responses. These practice procedures assured that subjects were aware of the proper temporal location of the prime stimulus in the stimulus sequence. The response window stimulus (exclamation point) was maintained so that the procedures for the prime visibility test would be as similar as possible to those of the already completed priming test.
After the two practice blocks came four data-collection blocks of 48 trials each, with prime duration restored to $33 \mathrm{~ms}$ and error feedback discontinued. Subjects continued to receive feedback reporting their latency and percent correct, with percent correct scored as the percent of trials for which the subject's response agreed with the prime stimulus's relation to 55

\section{Signal Detection Measures}

With the response window procedure, relatively high error rates occur, and the expectation is that pressing the left key versus the right key will often be under the control of the masked prime stimulus, rather than the visible target stimulus (Klinger, Burton, \& Pitts, 2000). The dependent measure is signal detection theory's sensitivity $\left(d^{\prime}\right)$ measure, computed by treating trials for which the prime stimulus is greater than 55 as signal trials and those for which the prime is less than 55 as noise trials. The hit rate is thus the proportion of right key responses to signal trials, and the falsealarm rate is the proportion of right key responses to noise trials.

Two such signal detection measures are of interest. For the last four trial blocks of the experiment, when the subject was trying to identify the prime stimulus, the computed $d^{\prime}$ measured the visibility of the prime stimulus. This is the direct measure, abbreviated $d_{\mathrm{dir}}^{\prime}$. For the five blocks of trials on which the subject was trying to respond to the target stimulus (and was typically not aware that a masked prime preceded the target), the $d^{\prime}$ sensitivity measure provided an indirect measure that indicates the priming effect, abbreviated $d_{\text {ind }}^{\prime}$. For both of these $d^{\prime}$ measures, the score reflects agreement of the subject's response with the visually masked prime stimulus, not the (visible) target stimulus.

\section{Results and Discussion}

\section{Preliminary Analyses}

Two important questions were to be answered from Experiment 1's data: First, were the prime stimuli effectively masked? And,

\footnotetext{
${ }^{2}$ The window center (interval between target onset and center of the response window interval-initially $400 \mathrm{~ms}$ ) was increased by $33 \mathrm{~ms}$ if, in the just finished block (a) the subject's error rate in classifying targets was $45 \%$ or greater, or (b) the error rate was at least $35 \%$ and the mean response latency was more than $100 \mathrm{~ms}$ greater than that block's window center value. The interval was decreased by $33 \mathrm{~ms}$ if the subject's error rate was $20 \%$ or lower and mean latency was not more than the window center value plus $100 \mathrm{~ms}$.
} 
second, did they produce priming? The simplest analyses to answer these questions looked at the overall average values of $d_{\mathrm{dir}}^{\prime}$ (the measure of prime visibility) and of $d_{\text {ind }}^{\prime}$ (the measure of priming). These tests were initially conducted with minimal selection of data (deleting $1.9 \%$ of indirect-measure trials that were slower than $800 \mathrm{~ms}$ and $0.1 \%$ of direct-measure trials that were slower than $5000 \mathrm{~ms}$ ). In this initial analysis, the mean of the prime visibility measure, $d_{\mathrm{dir}}^{\prime}$, was $.086(S D=.205)$. Although this value was statistically significantly greater than zero, $t(26)=2.19, p=$ .04 , still it was a very low value, indicating that the prime stimuli were quite effectively masked. For comparison, the mean value of the direct measure in the first experiment of Naccache and Dehaene (2001) was approximately $d_{\mathrm{dir}}^{\prime}=.6$. The mean value of the measure of priming, $d_{\text {ind }}^{\prime}$, was $.175(S D=.257)$, which was statistically significantly greater than zero, $t(26)=3.53, p=.002$.

Because the initial overall analysis left some ambiguity about whether the observed priming effect was associated with lack of visibility of prime stimuli, the regression analysis method of Greenwald, Klinger, and Schuh (1995) was applied. This regression analysis examines the intercept of the linear regression of the priming measure, $d_{\text {ind }}^{\prime}$, on the prime visibility measure, $d_{\text {dir- }}^{\prime}$ A statistically significant intercept effect in this regression analysis supports the interpretation that significantly above-zero priming on the indirect measure is associated with zero visibility on the direct measure. (Extensive discussion of this method and its interpretation can be found in Dosher, 1998; Greenwald \& Draine, 1998; Klauer, Draine, \& Greenwald, 1998; Klauer \& Greenwald, 2000; Klauer, Greenwald, \& Draine, 1998; Merikle \& Reingold, 1998; and Miller, 2000.) For Experiment 1's data, the regression analysis revealed a significant intercept, $d_{\text {ind }}^{\prime}=.154, t(26)=2.86, p=$ .009 , supporting the conclusion that priming effects were produced by masked primes that escaped visibility.

\section{Latency Operating Characteristic Analyses}

Because the priming effects observed in the analysis of overall means and in the regression analysis were small, the present data set provided a good opportunity to apply a recently developed method that has the potential to amplify evidence for priming. Using four sets of data obtained with the response window procedure, Abrams and Greenwald (2003) demonstrated that $d_{\text {ind }}^{\prime}$ is typically more than doubled, relative to its overall mean, when examined in a subset of trials selected from the fastest third of the overall distribution of latencies. Abrams and Greenwald's analysis was conducted by sorting responses by latency, then examining priming effects for about 50 subsets of responses that were grouped by speed, ranging from fastest (lowest percentiles of the distribution) to slowest (highest percentiles). Their four data sets all showed the same pattern, with priming effects (i.e., $d_{\text {ind }}^{\prime}$ ) (a) being maximal in approximately the 20th to 30th percentile, (b) steadily declining for responses slower than the 30th percentile, and (c) remarkably, becoming negative for responses slower than about the 60th percentile. These patterns made clear that statistical sensitivity to priming effects is enhanced by examining trials from faster portions of the latency distribution. The most complete picture of the data is provided by the full latency operating characteristic (LOC) function (cf., Lappin \& Disch, 1972, 1973).

Figure 2A shows the LOC analysis of Experiment 1's priming (indirect-measure) data. This LOC curve is accompanied by a $99.5 \%$ confidence interval. Where the lower bound of the confidence interval is above zero, the priming effect is significantly greater than zero at a very conservative two-tailed $\alpha=.005$ criterion. Figure $2 \mathrm{~A}$ reveals (a) maximum priming at latencies below $400 \mathrm{~ms}$, and (b) magnitude of priming dropping below zero for latencies above $500 \mathrm{~ms}$. These two characteristics of the LOC in Figure 2A are not unique to the data of present Experiment 1. Both characteristics appeared in each of the four sets of data analyzed by Abrams and Greenwald (2003). The elevated priming in the faster portion of the LOC is typical of the manner in which the LOC analysis makes priming effects more sharply evident.

Direct-measure results are presented in Figure 2B. This linear LOC is accompanied by a $95 \%$ confidence interval that shows no statistically significant deviation from zero. ${ }^{3}$ The combination of tests provided by the two LOCs in Figure 2 again support the conclusion that significant priming was produced by masked stimuli that were essentially invisible. Note that the estimated magnitude of $d_{\text {ind }}^{\prime}$ associated with latencies of $300 \mathrm{~ms}$ or less in Figure $2 \mathrm{~A}$ is about .6 , more than triple the overall mean value of $d_{\text {ind }}^{\prime}$.

\section{Additional Hypothesis Tests}

All of Experiment 1's priming stimuli were two-digit numbers that had never appeared as visible stimuli in the subject's task of classifying quantity relative to 55 . Among these new-set stimuli were two subsets of primes that might be expected to produce different priming effects. Half of the primes were composed of two digits that were either both less than 5 or both greater than 5 (these were homogeneous-digit primes: e.g., 14, 22, 32, 41, 66, 78, 88, 96). For the remainder, the two digits were on opposite sides of 5 (heterogeneous-digit primes: e.g., 16, 27, 38, 49, 61, 73, 82, 94). From previous findings that subliminal priming effects are produced by components of multiletter primes (Abrams \& Greenwald, 2000), it was plausible that subliminal priming effects of two-digit numbers might be produced by their single-digit components. This reasoning leads to the expectation that the homogeneous-digit primes might show larger priming effects. However, this was not the case. Examination of overall effects on $d_{\text {ind }}^{\prime}$ (and also of LOCs) indicated no difference in effects for these two sets of primes.

An additional analysis tested for differences in priming for the two counterbalancing conditions-one with primes constructed entirely from the digits $1,4,6$, and 9 , and the other with primes constructed using only 2, 3, 7, and 8. Although the overall and LOC methods both indicated that priming effects were stronger for the latter set, this difference was not close to being statistically significant.

\footnotetext{
${ }^{3}$ When a curvilinear (cubic) regression function was fitted to the $d_{\mathrm{dir}}^{\prime}$ data in Figure 2B, there was a statistically significant elevation above zero for a $60-\mathrm{ms}$ wide range of latencies between 300 and $400 \mathrm{~ms}$. This above-chance performance on the direct measure was possibly due to the same automatic priming effect shown in Figure 2A. Recall that subjects had been instructed to respond slowly on this task because of the experimenters' concern that rapid responding would convert the prime-visibility task into another priming measure. That is, if subjects responded rapidly on the prime-visibility task they might show elevated performance due to unconscious priming rather than due to conscious perception of the primes. Subjects who ignored the instruction to respond slowly may have done so in order to shorten a task that was frustrating because of their inability to perceive the masked stimuli.
} 
Experimental task: Classify 2-digit numbers as less-than-55 or greater-than-55

Visible targets: $11,14,15,16,19,41,44,45,46,49,51,54$ - 56,59,61,64,65,66,69,91,94,95,96,96

Masked primes: $27,28,37,38,22,23,32,33$ - 72,73,82,83,77,78,87,88

A

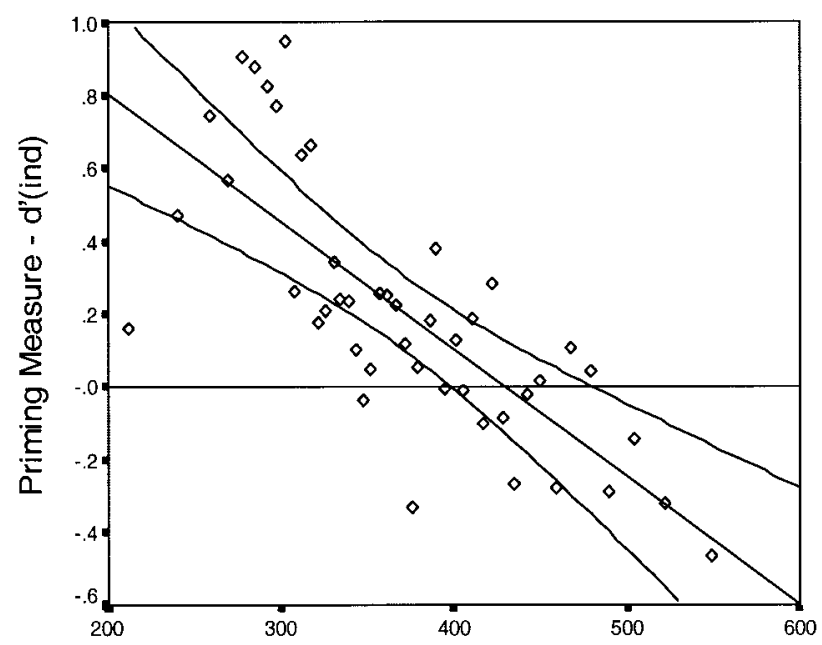

B

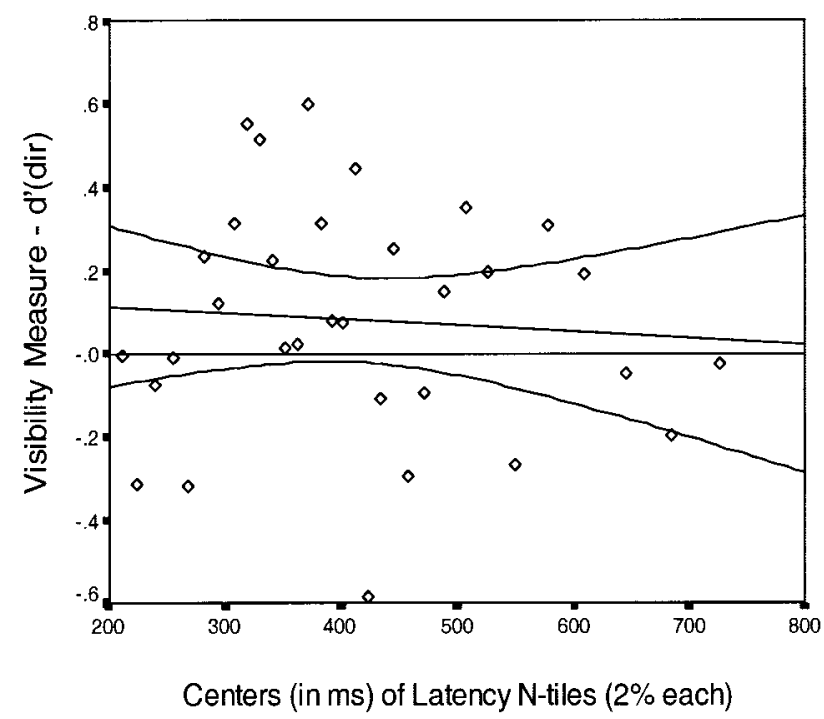

Figure 2. Linear regression (LOC) plots for sensitivity to masked primes as a function of response latency. A: Indirect measure, showing influence of the prime when the task is to classify the immediately $(67 \mathrm{~ms})$ following visible target stimulus. B: Direct measure, showing sensitivity to the prime when subjects were trying to see and classify it. Both plots treat the entire experiment's data as those of a single subject. The upper regression plot is flanked by boundaries of its $99.5 \%$ confidence interval, the lower one by $95 \%$ confidence interval boundaries. Data from Experiment 1: $N=27$.

In summary, Experiment 1's results for new-set primes were consistent with Naccache and Dehaene's (2001) conclusion that visually masked numbers receive analyses that have access to long-term semantic memory. The critical finding was that masked priming effects occurred for number stimuli that (a) were not visible to subjects, and (b) had never been presented as visible targets in the task of classifying numbers as smaller or greater than 55. This pattern of findings was in contrast to the absence of subliminal priming in previous similar experiments using new-set word stimuli in a valence classification task (Abrams \& Greenwald, 2000, Experiment 3).

\section{Experiment 2}

Experiment 1 confirmed Naccache and Dehaene's (2001) finding of subliminal priming that could be attributed to long-term semantic memory of numbers. With this achieved, Experiment 2 set out to examine more closely the properties of unconscious analysis responsible for priming effects by number stimuli. In Experiment 1, the two digits of each masked prime were new set, meaning that they had not appeared in visible targets and, therefore, their priming effects must have resulted from access to long-term semantic memory. In Experiment 2 only the first digits 
of masked primes had this property. The stimulus design of Experiment 2 (see Table 2) incorporated independent variables that are described in the next two paragraphs.

\section{Attention to First Versus Second Digit Position}

For Experiment 1, because no masked primes had a first digit of 5, priming effects could have resulted from unconscious analysis of just the first digits of primes, which provided sufficient information to classify the prime as smaller or larger than 55 . Also, 20 of Experiment 1's 24 old-set (visible target) stimuli could be responded to correctly by attending just to their first digits. Possibly, therefore, the priming effect in Experiment 1 was caused by or facilitated by subjects having learned to attend primarily to the first digits of the experimental stimuli. Experiment 2 tested the possible effect of such task-induced attentional focus by varying the visible target stimuli. For half the subjects, the visible targets obliged attention to the first digit because the second was an uninformative 5; for the remainder, the visible targets obliged attention to the second digit because the first digit was a 5 .

\section{Nonparadoxical Versus Paradoxical Primes}

Abrams and Greenwald (2000) introduced the notion of paradoxical primes. These are primes for which contextual meaning opposes long-term semantic meaning by virtue of their being composed of parts of practiced, visible target words that had opposite valence (e.g., tumor as a prime constructed from parts of the practiced targets tulip and humor). Half of the number primes in Experiment 2 were paradoxical, in this sense, because their second digits had been encountered in practiced, visible targets from the opposite quantity category. For example, the masked prime 16 (bottom left cell of Table 2) was paradoxical because long-term semantic meaning classifies it as less than 55, but its second digit (6) might have acquired opposed contextual memory associations due to practice in classifying either 56 or 65 as greater than 55 .

\section{Method}

\section{Subjects}

Forty University of Washington undergraduate students participated in exchange for credit toward a course requirement. All had normal or corrected-to-normal vision, were fluent in English, and were naive about the hypothesis of the experiment.

\section{Procedure}

Experiment 2 repeated the basic structure of Experiment 1, including the sequence of (a) practice (with no masked primes) at the task of classifying two-digit numbers, (b) a priming test that obtained the indirect measure data, and (c) a prime visibility test that obtained the direct measure data.

Subjects classified only six different visible two-digit numbers and received priming trials that included only 12 different masked primes (see Table 2). Because Experiment 2 used substantially fewer target and prime stimuli than Experiment 1, the total number of trials in the experiment was reduced. The third and fourth practice blocks were reduced from 48 to 24 trials each, and the masked priming task included four (rather than five) blocks of 48 trials. The concluding prime visibility task was unchanged, including two 48-trial practice blocks (with lengthened prime durations) before four blocks of 48 data collection trials.

\section{Results}

Results were analyzed in the same fashion as for Experiment 1, examining first the overall means for $d_{\text {ind }}^{\prime}$ and $d_{\text {dir }}^{\prime}$, then the intercept in the regression of $d_{\text {ind }}^{\prime}$ on $d_{\mathrm{dir}}^{\prime}$, and last, LOC analyses in which the priming and visibility $d^{\prime}$ measures were examined as a function of response latency. As was the case for Experiment 1, all methods of analysis were consistent with one another. Also as for Experiment 1, the clearest picture of the results was given by the LOC analyses, for which results are presented in Figure 3, and only these results are described here.

\section{Tests for Prime Visibility}

All tests on $d_{\text {dir }}^{\prime}$ indicated that the primes were not visible. Overall means did not differ significantly from zero, and examination of the $95 \%$ confidence intervals of the LOC functions (such as in the lower panel of Figure 2B) for each category of primes revealed no indications of any deviation from zero visibility associated with any portions of these functions.

\section{Effects of Digit-Position Attentional Focus and Paradoxicality}

The effects of attentional focus were strong. When the visible task obliged attention to first digits, masked-priming effects appeared to be controlled by the primes' first digits, even though those digits had never appeared in the set of visible targets. These

Table 2

Stimulus Design for Experiment 2

\begin{tabular}{lll}
\hline Stimulus and prime & Numbers less than 55 & Numbers greater than 55 \\
\hline Visible target stimuli $^{\mathrm{a}}$ & & \\
Focus on digit $1^{\text {Focus on digit 2 }}$ & $25,35,45$ or $15,25,35$ & $65,75,85$ or $75,85,95$ \\
Masked primes & $52,53,54$ or $51,52,53$ & $56,57,58$ or $57,58,59$ \\
$\quad$ Nonparadoxical & $12,13,14$ or $41,42,43$ & $96,97,98$ or $67,68,69$ \\
Paradoxical & $16,17,18$ or $47,48,49$ & $92,93,94$ or $61,62,63$ \\
\hline
\end{tabular}

Note. Half of the subjects were in each digit focus condition and, within each of those groups of subjects, half were in each counterbalancing condition, corresponding to the stimuli listed to the left or right in each cell. Subjects received exclusively either the stimuli shown to the left or those shown to the right.

a Positional focus is a between-subjects variable. ${ }^{\mathrm{b}}$ Paradoxicality is a within-subjects variable. 
Visible Task Requires Focus on Digit $1(25,35,45$ vs. $65,75,85)$
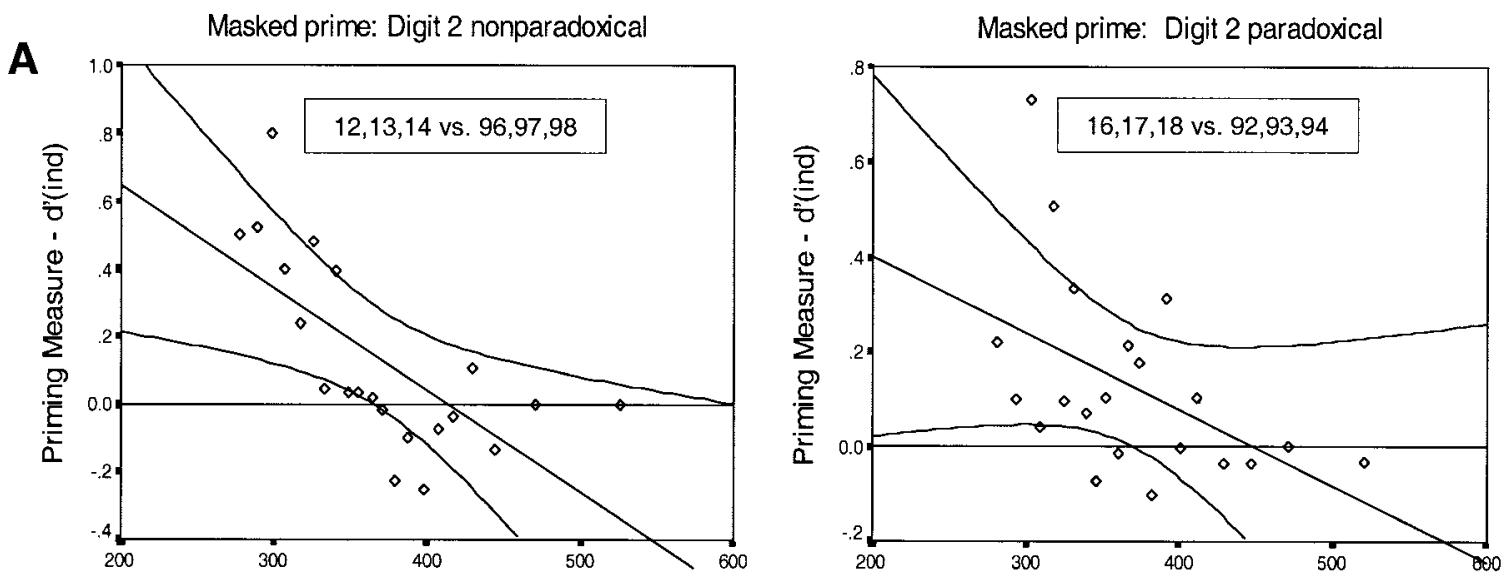

Visible Task Requires Focus on Digit $2(52,53,54$ vs. $56,57,58)$
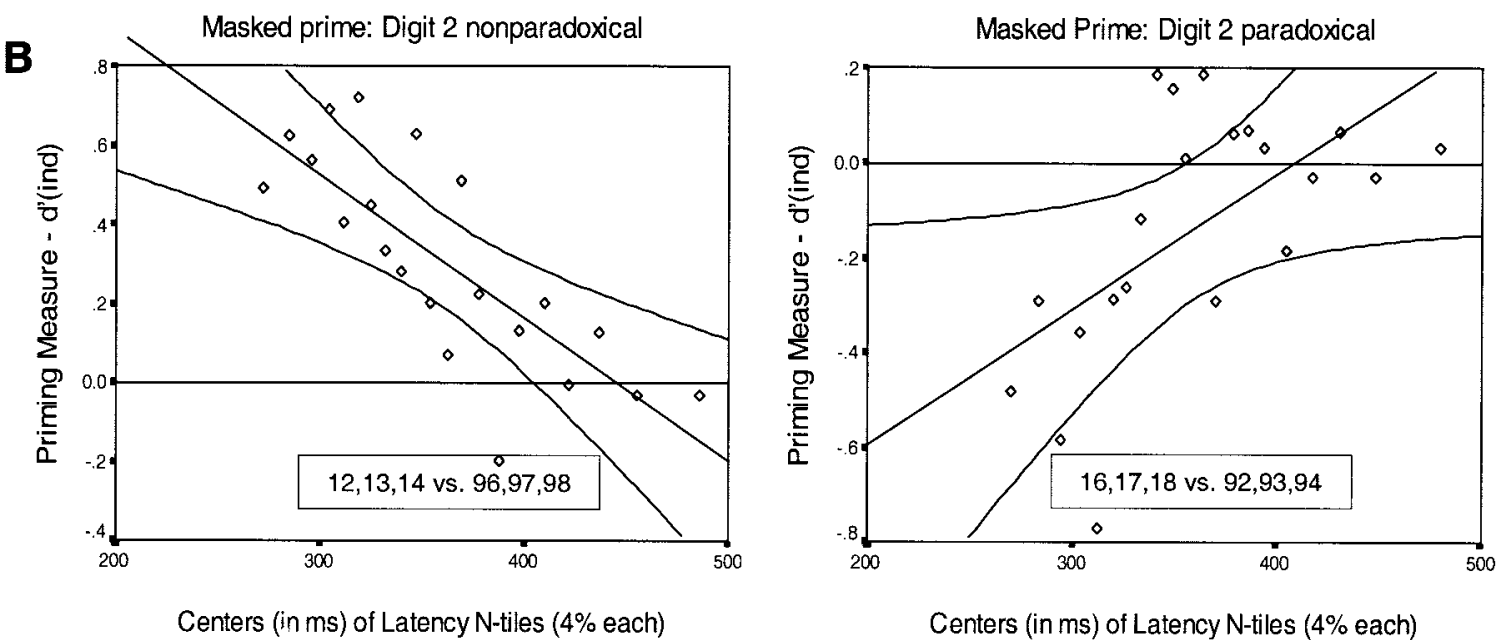

Figure 3. Linear regression (LOC) plots for priming conditions in Experiment $2(N=40)$. Paradoxical prime digits were ones that had been seen by the subject only in visible targets that were smaller than 55 but appeared in a prime that was greater than 55, or vice versa. See text for further explanation.

results are shown in Figure 3A. Both of these LOCs revealed clear priming effects at latencies below $400 \mathrm{~ms}$. Also, these two priming effects were quite similar-meaning that it made little difference whether or not the second digit was paradoxical (compare the two panels of Figure 3A). Thus, when the visible task focused attention on the first digit, priming appeared to be based on long-term semantic memory, with no indication of an effect of contextual memory. Had there been a contextual memory effect of the second digit, then stimuli that were paradoxical (because the contextual meaning of their second digits opposed the long-term semantic meaning of the two-digit string) should have yielded weaker or even reversed priming effects. Although priming effects were slightly reduced when the second digit was paradoxical (compare confidence interval boundaries for the two panels of Figure 3A), there were no statistically noteworthy differences associated with this comparison.

By contrast, when the visible task obliged attention to second digits, masked-priming effects appeared to be controlled by the primes' second digits, as shown by a reversed priming effect when second digits were paradoxical. This result is clearly apparent in comparing the two panels of Figure 3B. The priming effect in each case was the effect expected if the prime's second digit tended to elicit the response based on its contextual memory associations. When the second digit of the prime was nonparadoxical (i.e., it had been seen in visible numbers that had the same relation to 55 as did the prime) the expected congruency-priming effect was clearly apparent in the faster-than-400-ms portion of the LOC function (Figure 3B, left panel). However, when the prime's second digit was paradoxical (i.e., contextual memory associations opposing long-term semantic memory associations), there was an equally clear reversed priming effect (Figure 3B, right panel), indicating the dominance of the second digit's contextual memory associations.

Experiment 2's priming effects, shown in Figure 3, can be summarized in two conclusions. First, the attentional manipulation had a strong effect: Priming effects were carried by the digit in the position on which subjects had been obliged to focus. Second, when the visible task obliged attention to the first digit, priming 
effects were based on long-term semantic memory-these were effects of digits that were never encountered in the visible target set and, therefore, their effects could be based only on long-term semantic memory.

Overall, Experiment 2 provided a second clear confirmation of Naccache and Dehaene's (2001) finding, for the condition in which the visible task obliged focus on the first digit. At the same time, when the visible task obliged attention to the second digit, there was no indication that prime meaning based on long-term semantic memory had any effect.

\section{Discussion}

The results of Experiment 2 allow a sharpening of Experiment 1 's conclusions. Most important was the finding of priming by numbers not included among the visible stimuli for the quantity classification task. This replicated Experiment 1 and again confirmed Naccache and Dehaene's (2001) finding of a similar effect based on long-term semantic memory associations of numbers. Experiment 2 also showed that digit-position focus induced by the task for visible target numbers extended to the analysis of masked primes. For example, when the task for visible numbers obliged a focus on the second digit, only the second digits of masked primes were effective in priming. Finally, Experiment 2 revealed a paradoxical masked-priming effect that occurred only when the task for visible targets required subjects to focus on second digits (Figure 3B, right panel).

A simple summary of Experiment 2's findings is that long-term semantic memory dominated unconscious analysis of masked primes when the visible task focused attention on first digits, whereas contextual memory dominated when the task focused attention on second digits. Because the very same pairs of primes and targets were used for the priming test in both cases, this implies that subliminal priming effects can be strongly modulated by the task context.

\section{Experiment 3}

In combination, Experiments 1 and 2 demonstrated unconscious retrieval of long-term semantic meaning for visually masked numbers that had not been experienced as visible targets in the experiment. Experiment 2 additionally showed that masked priming effects of two-digit numbers were produced by the digit in the position (first or second) to which attention was directed by the target stimuli for the quantity classification task.

Experiment 3 further explored the role of attention to digits by asking whether it is possible to induce subjects to process both digits of a masked two-digit numeral. In this experiment, the target stimuli were selected so that those smaller than 55 were composed of a distinctive set of digits (e.g., numbers 16, 19, 46, 49), whereas those larger than 55 were composed of a different set of digits (e.g., numbers 72, 73, 82, 83). To assess the effect of this task context on processing of masked primes, subjects were later tested with novel primes that shared either the left digit (e.g., 15, 25), or both digits (e.g., 27) with the practiced target set. If unconscious analysis is based solely on contextual memory of individual digits rather than on long-term semantics, a masked prime such as 27 should now paradoxically prime the "larger" response because both of its digits had been consistently associated with visible targets that were larger than 55 (i.e., 72, 73, 82, or 83).

\section{Method}

\section{Subjects}

Thirty-five University of Washington undergraduate students participated in exchange for credit toward a course requirement. All had normal or corrected-to-normal vision, were fluent in English, and were naive about the hypothesis of the experiment.

\section{Procedure}

Procedures were nearly identical to those of Experiment 1 . The only difference was in the selection of stimuli used as visible targets and as masked primes. The stimulus design for Experiment 3 appears in Table 3.

Experiment 3's design included visible stimulus sets that obliged attention to the first digit. It also included three categories of masked primes: Nonparadoxical primes contained first digits that had been encountered in visible target stimuli, and these digits were unchanged in position. Paradoxical masked primes had first digits that had been encountered as second digits of visible targets that had the opposite relation to 55. These should show paradoxical (i.e., reversed) priming only if the contextual memory effect of the changed-position digit was stronger than its long-term semantic memory effect. Doubly paradoxical masked primes contained two digits that had been encountered in targets with the opposite relation to 55, and both of these digits were changed in position from the positions in which they had been experienced in visible targets. These again tested the possibility that masked priming effects due to contextual associations could

Table 3

Stimulus Design for Experiment 3

\begin{tabular}{lcc}
\hline Stimulus and prime & Numbers less than 55 & Numbers greater than 55 \\
\hline Visible target stimuli & $16,19,46,49$ or & $72,73,82,83$ or \\
& $27,28,37,38$ & $61,64,91,94$ \\
Masked primes $^{\mathrm{a}}$ & 15,45 or 25,35 & 75,85 or 65,95 \\
Nonparadoxical & 25,35 or 15,45 & 65,95 or 75,85 \\
Paradoxical & $27,28,37,38$ or & $61,64,91,94$ or \\
Doubly paradoxical & $16,19,46,49$ & $72,73,82,83$ \\
\hline
\end{tabular}

Note. In the counterbalancing design, half of the subjects received exclusively either the stimuli shown to the left or to the right in each cell. That is, for example, subjects who saw 16, 19, 46, 49 as less-than-55 visible targets also received, as less-than-55 masked primes, 15, 45, 25, 35, 27, 28, 37, 38.

${ }^{a}$ Paradoxicality is a within-subjects variable. 
occur for stimuli changed in position from the visible stimuli in which they had acquired those contextual associations.

\section{Results}

\section{Priming}

Figure 4 presents the LOC functions separately for the three categories of primes used in Experiment 3. The data for nonparadoxical primes (Figure 4A) showed a substantial priming effect concentrated in the faster half of the data. Data for the paradoxical primes (Figure 4B) showed no evidence of priming, while those for the doubly paradoxical primes (Figure 4C) showed evidence for reversed priming in the faster half of the LOC. As can be seen in Figure 4, the reverse effect for doubly paradoxical primes was noticeably smaller in magnitude than the (proper direction) effect for nonparadoxical primes, and the shapes of these two functions were approximate mirror images of each other throughout their respective ranges.

\section{Prime Visibility}

The direct measure data for both nonparadoxical and paradoxical primes showed no statistically significant departures from $d_{\mathrm{dir}}^{\prime}=0$ at any point on their LOC curves. Only the LOC curve for the doubly paradoxical primes, when fit with a cubic regression function, showed a significant positive departure from $d_{\text {dir }}^{\prime}=0$, and this departure was only for a small (60-ms wide) segment of the LOC. That small departure from zero visibility for the doubly paradoxical masked primes is probably not meaningful, given that it was opposite in direction to the effect expected if subjects could see these primes.

\section{Discussion}

Experiment 3 extended Experiment 2's finding of a paradoxical masked-priming effect due to contextual associations acquired during the experiment. In Experiment 3, paradoxical maskedpriming effects were produced by digits that were switched from the first position of visible targets to the second position of masked primes. The magnitude of this effect can be seen in the comparison of Figure 4B and 4C. By contrast, in the Digit-1-Focus condition of Experiment 2, digits that had undergone the same position switch showed no paradoxical effect (compare the two panels of Figure 3A).

This contrast between findings of Experiment 3 and Experiment 2's Digit-1-Focus condition prompts a search for the difference between the two experiments that might have caused these different patterns. Almost certainly, the critical difference was that the visible targets of Experiment 2's Digit-1-Focus condition always had 5 as the second digit. This meant that the digit in the second position had no relation at all to the required $(<55$ or $>55)$ response. By contrast, the second digits of visible targets in Experiment 3-although in principle useless for the required discrimination (because the first digit was never a 5)-were perfectly correlated with the required response. Thus, subjects apparently learned the usefulness of second-position digits, and then showed a strong paradoxical priming effect due to digits in second position of masked primes, which were digits that had never appeared in the second position of visible targets.
Experiment 3 also provided evidence for effects of digits that had changed position from the second position of targets to the first position of masked primes (i.e., the first digits of the paradoxical masked primes in Figure 4B). If these digits had acquired no contextual associations, then the data pattern in Figure 4B should have shown a priming effect resembling those in Figure 2A or in Figure 3A, both of which display data from conditions in which the first digits of masked primes could not have acquired contextual associations. To the contrary, however, Figure 4B shows no priming at all, plausibly indicating a balance between effects due to long-term semantic associations and those due to contextual associations of the changed-position digits.

The results of Experiment 3 can be summarized by noting that, in a situation in which the separate, individual digits of the target numbers were consistently associated with response categories, priming was entirely dependent on the digits taken separately. Perhaps, in Experiment 3, because of the perfect association of individual digits with required responses, the task context induced subjects to process the target numbers in a rote fashion without regard to their numeric meaning. For example, in one of the two counterbalancing conditions, digits $1,4,6$, and 9 appeared only in targets smaller than 55, while digits $2,3,7$, and 8 appeared only in targets greater than 55 . The hypothesized rote-processing orientation might then have generalized to the two-digit primes. As a check on whether subjects processed the two-digit targets in rote fashion rather than in terms of their semantic (numeric) values, the data of Experiment 3 were examined to see if they displayed the robust numeric distance effect that was described by Hinrichs, Yurko, and Hu (1981; see also Dehaene, Dupoux, \& Mehler, 1990). This numeric distance effect takes the form of superior responding the more distant the target number is from the standard (e.g., relative to the standard of 55, 19 is more distant than 46, such that it should be easier to classify 19 than 46). In Experiment 3, this effect should appear in error rates rather than latencies, as a consequence of the forced rapid responding of the response window procedure. The expected numeric distance effect was clearly apparent. There was a $17 \%$ error rate for the distant values $(16,19$, 91, and 94) compared to a $25 \%$ rate for the close values (46, 49, 61 , and 64); $t(34)=3.84, p=.0005$. Closer examination showed that this error difference increased through the five blocks of the priming task. These observations were consistent with concluding that Experiment 3's visible target numbers were processed semantically, rather than merely in rote fashion.

\section{General Discussion Review of Findings}

Experiments 1 and 2 found that visually masked numbers receive analyses that make contact with long-term semantic memory of number meanings. Their methods and results add confidence to Naccache and Dehaene's (2001) previous conclusion that such analysis of visually masked numbers can occur unconsciously. The chief added features of the present research were use of a regression method (Greenwald et al., 1995) to demonstrate occurrence of priming effects when primes are not visible and use of latency operating characteristic (LOC) analyses to amplify evidence for masked-priming effects.

Experiments 2 and 3 applied Experiment 1's methods to demonstrate, in several ways, that subliminal priming by visually 
Visible Targets: $(16,19,46,49 \cdot 72,73,82,83)$

A

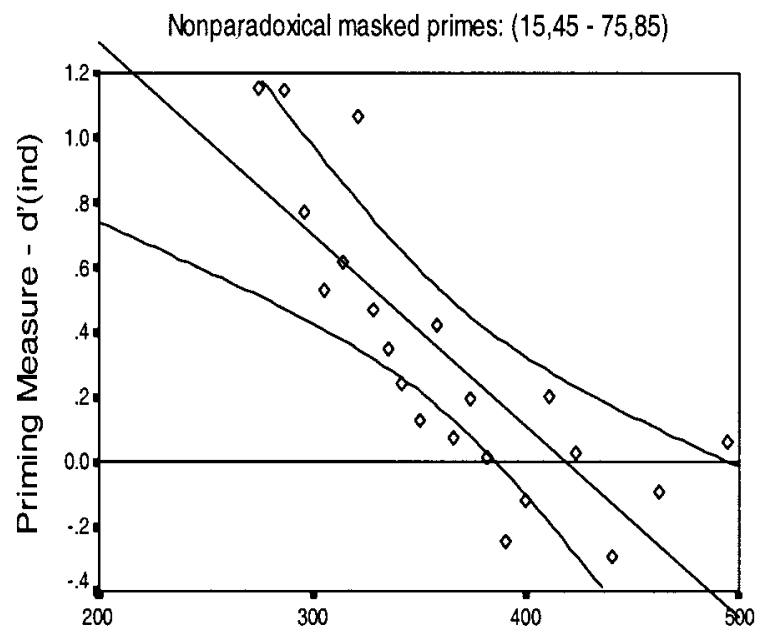

Paradoxical masked primes: $(25,35-65,95)$

B

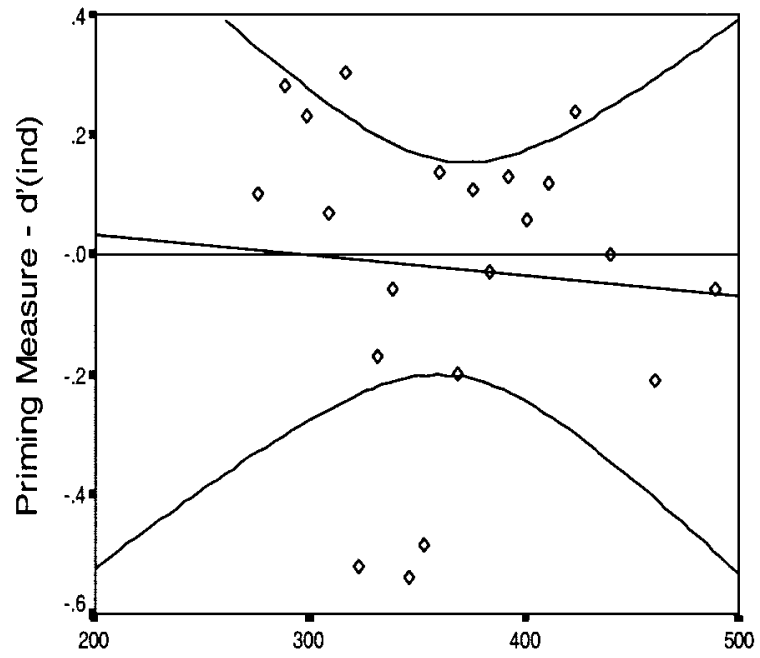

Doubly paradoxical masked primes: $(27,28,37,38-61,64,91,94)$

C

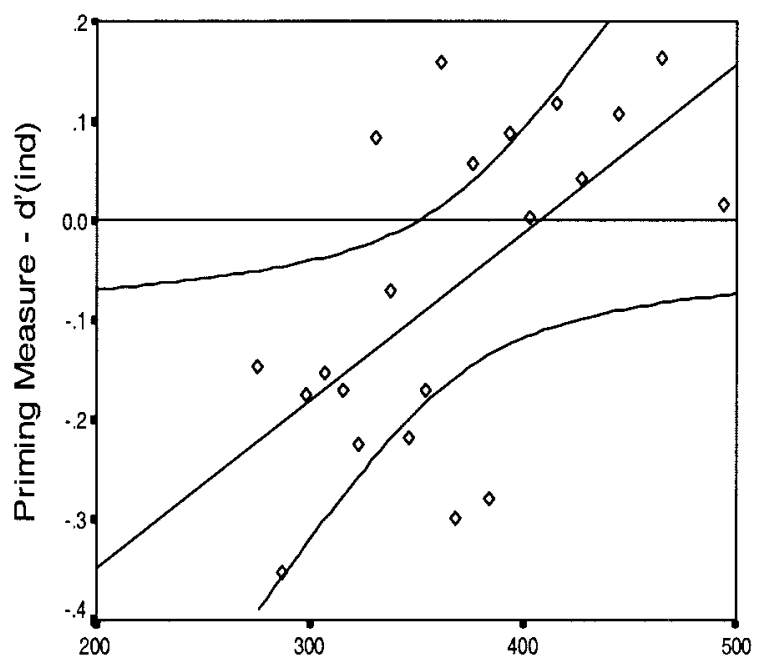

Centers (in ms) of Latency N-tiles (4\% each) masked numbers is sensitive to contextual memory resulting from the subject's practice experiences in the experiment. In Experiment 2, after practice that obliged focus on either the first or second digit position, masked-priming effects were carried almost entirely by the prime digit position on which attention had been focused in practice. Experiments 2 and 3 both demonstrated paradoxical priming effects that caused two-digit numbers to function as primes in a fashion that disagreed with their actual numeric value. For example, in Experiment 2, after practice in which the digit 6 was seen only in classifying 56 as greater than 55 , the masked prime 16 facilitated the greater-than-55 response (Figure 3B, right).

\section{Dominance of Contextual Memory Over Long-Term Semantic Memory in Masked Priming}

Relatively few masked-priming studies have provided the opportunity to observe effects of long-term semantic memory alongside effects of memories established within the experiment (contextual memory). Abrams and Greenwald (2000) found only contextual memory effects, as did Damian (2001). In both of those studies, words not practiced as visible targets were ineffective as masked primes. Klinger, Burton, and Pitts (2000), using words that could be classified both by animacy (living vs. nonliving) and by affect (pleasant vs. unpleasant), found that masked-priming effects occurred only for the dimension on which subjects had practiced classifying the words. Only Naccache and Dehaene (2001) and the present research, using number stimuli, found evidence for priming based on long-term semantic memory. Out of four tests in the present research in which long-term memory and contextual memory effects were put in opposition, long-term memory dominated in only one (Figure 3A, right), the effects were balanced in another (Figure 4B), and contextual memory proved stronger in two (Figure $3 \mathrm{~B}$, right, and Figure $4 \mathrm{C}$ ).

Across all relevant studies, then, contextual memory effects on masked priming have tended to dominate those based on long-term memory. None of the available evidence suggests why this might be so. Interestingly, this dominance of contextual memory indicates that the unconscious form of memory observed in these studies has the flexibility to adapt to recent experience.

\section{Differences Between Numbers and Words?}

The present research demonstrated access to long-term semantic memory by number stimuli that were visually masked to reduce their measured visibility to zero or near zero. This confirmed a finding previously reported by Naccache and Dehaene (2001). In other research that used similar conditions to test access to longterm semantic memory for words, such access to long-term semantic memory has not been found (Abrams \& Greenwald, 2000, Experiment 3). ${ }^{4}$ The use of parallel procedures in these studies with words and numbers suggests that the differences in results

Figure 4. Linear regression (LOC) plots for priming conditions in Experiment $3(N=35)$. Doubly paradoxical primes were ones for which both digits had been seen by the subject only in visible targets that had the opposite relation to 55 , such as visible 73 followed by use of 37 as a prime. 
must be due to differences in how number and word stimuli are processed.

Numbers are among the most frequent content words of any language, and the frequency of Arabic numerals is even higher than that of spelled-out number words (Dehaene \& Mehler, 1992). Thus, semantic access to number meaning may be especially fast and easy. Several experiments have demonstrated fast and automatic activation of number meanings in tasks for which number meaning was clearly irrelevant (Brysbaert, 1995; Dehaene \& Akhavein, 1995; Dehaene, Bossini, \& Giraux, 1993; Fias, Brysbaert, Geypens, \& d'Ydewalle, 1996). The Arabic-numeral format of numbers used in the present experiments may be especially efficient as cues for retrieving long-term semantic representations (Dehaene, 1996; Dehaene et al., 1998; Pinel, Rivière, Le Bihan, \& Dehaene, 2001).

Most numbers have a single, well-defined meaning: the quantity that they represent. This contrasts with many high-frequency words, which have multiple aspects of meaning. The studies that have not obtained subliminal semantic priming with words are ones that required subjects to extract a single aspect of meaning from semantically multifeatured words, for example, extracting positive or negative valence (Abrams \& Greenwald, 2000) or physical size of the object represented by a word (Damian, 2001). The finding that these semantic features are not accessed subliminally need not imply that semantic features of words are generally excluded from unconscious analyses.

An additional consideration is that time pressure may have different effects in processing number and word stimuli. The present research used the same response window of $400 \mathrm{~ms}$ that had been used in parallel experiments with words. If semantic access is faster for numbers than for other words, this procedure may create different time pressures on number and word decisions. It is relatively easy to compare numbers within $400 \mathrm{~ms}$, even in the absence of a response deadline (e.g., Dehaene, 1996). Responding so rapidly to discriminations of word meaning may be much more difficult. In order to meet the response deadline, subjects in the Abrams and Greenwald (2000) experiment may have been forced to adopt a shallow strategy, basing their responses on word fragments rather than on semantics.

Finally, a potentially important difference is that, unlike single letters, single-character numbers have unambiguous semantic meaning. The finding that visually masked numbers (but not words) receive semantic analysis conceivably follows from this reliable association of single digits, but not single letters, with semantic meaning. Therefore, it may be worth doing further experiments in which word stimuli are made as comparable as possible to the number stimuli of the present research both in frequency of occurrence (high) and stimulus length (short).

\footnotetext{
${ }^{4}$ To confirm the nonfinding with word stimuli, a new replication of Abrams and Greenwald's (2000) Experiment 3 was conducted and analyzed using the LOC methods of the present research. This new experiment with word stimuli is not presented in detail here, to conserve space. The LOC function obtained for its indirect (priming) measure was flat, entirely unlike the sharply, negatively sloped function of the comparable experiment with number stimuli in Figure 2A.
}

\section{References}

Abrams, R. L., \& Greenwald, A. G. (2000). Parts outweigh the whole (word) in unconscious analysis of meaning. Psychological Science, 11, $118-124$.

Abrams, R. L., \& Greenwald, A. G. (2003). A Latency-Based Strategy for Enhancing the Sensitivity of Accuracy Measures of Unconscious Semantic Priming. Manuscript submitted for publication.

Bodner, G. E., \& Masson, M. E. J. (2001). Prime validity affects repetition priming: Evidence for an episodic resource account of priming. Journal of Memory and Language, 616-647.

Brysbaert, M. (1995). Arabic number reading: On the nature of the numerical scale and the origin of phonological recoding. Journal of Experimental Psychology: General, 124, 434-452.

Damian, M. F. (2001). Congruity effects evoked by subliminally presented primes: Automaticity rather than semantic processing. Journal of Experimental Psychology: Human Perception and Performance, 27, 154165.

Dehaene, S. (1996). The organization of brain activations in number comparison: Event-related potentials and the additive-factors methods. Journal of Cognitive Neuroscience, 8, 47-68.

Dehaene, S., \& Akhavein, R. (1995). Attention, automaticity, and levels of representation in number processing. Journal of Experimental Psychology: Learning, Memory, and Cognition, 21, 314-326.

Dehaene, S., Bossini, S., \& Giraux, P. (1993). The mental representation of parity and numerical magnitude. Journal of Experimental Psychology: General, 122, 371-396.

Dehaene, S., Dupoux, E., \& Mehler, J. (1990). Is numerical comparison digital? Analogical and symbolic effects in two-digit number comparison. Journal of Experimental Psychology: Human Perception \& Performance, 16, 626-641.

Dehaene, S., \& Mehler, J. (1992). Cross-linguistic regularities in the frequency of number words. Cognition, 43, 1-29.

Dehaene, S., Naccache, L., Le Clech, G., Koechlin, E., Mueller, M., Dehaene-Lambertz, G., et al. (1998). Imaging unconscious semantic priming. Nature, 395, 597-600.

Dell'Acqua, R., \& Grainger, J. (1999). Unconscious semantic priming from pictures. Cognition, 73(1), B1-B15.

Dosher, B. A. (1998). The response-window regression method-Some problematic assumptions: Comment on Draine and Greenwald (1998). Journal of Experimental Psychology: General, 127, 311-317.

Draine, S. C., \& Greenwald, A. G. (1998). Replicable unconscious semantic priming. Journal of Experimental Psychology: General, 127, 286303.

Fias, W., Brysbaert, M., Geypens, F., \& d'Ydewalle, G. (1996). The importance of magnitude information in numerical processing: Evidence from the SNARC effect. Mathematical Cognition, 2, 95-110.

Greenwald, A. G., \& Draine, S. C. (1998). Distinguishing unconscious from conscious cognition-Reasonable assumptions and replicable findings: Reply to Merikle and Reingold (1998) and Dosher (1998). Journal of Experimental Psychology: General, 127, 320-324.

Greenwald, A. G., Draine, S. C., \& Abrams, R. L. (1996). Three cognitive markers of unconscious semantic activation. Science, 273, 1699-1702.

Greenwald, A. G., Klinger, M. R., \& Schuh, E. S. (1995). Activation by marginally perceptible ("subliminal") stimuli: Dissociation of unconscious from conscious cognition. Journal of Experimental Psychology: General, 124, 22-42.

Hinrichs, J. V., Yurko, D. S., \& Hu, J. (1981). Two-digit number comparison: Use of place information. Journal of Experimental Psychology: Human Perception \& Performance, 7, 890-901.

Holender, D. (1986). Semantic activation without conscious identification in dichotic listening parafoveal vision and visual masking: A survey and appraisal. Behavioral and Brain Sciences, 9, 1-23.

Inquisit 1.28 [Computer software]. (1998). Seattle, WA: Millisecond Software.

Klauer, K. C., Draine, S. C., \& Greenwald, A. G. (1998). An unbiased 
errors-in-variables approach to detecting unconscious cognition. British Journal of Mathematical and Statistical Psychology, 51, 253-267.

Klauer, K. C., \& Greenwald, A. G. (2000). Measurement error in subliminal perception experiments: Simulation analyses of two regression methods-Comment on Miller (2000). Journal of Experimental Psychology: Human Perception and Performance, 26, 1506-1508.

Klauer, K. C., Greenwald, A. G., \& Draine, S. C. (1998). Correcting for measurement error in detecting unconscious cognition: Comment on Draine and Greenwald (1998). Journal of Experimental Psychology: General, 127, 318-319.

Klinger, M. R., Burton, P. C., \& Pitts, G. S. (2000). Mechanisms of unconscious priming: I. Response competition, not spreading activation. Journal of Experimental Psychology: Learning, Memory, and Cognition, 26, 441-455.

Lachter, J., Forster, K. I., \& Ruthruff, E. (2000, November). Unattended words are not identified. Paper presented at the 41st Annual meeting of the Psychonomic Society, New Orleans, LA.

Lappin, J. D., \& Disch, K. (1972). Latency operating characteristic: I. Effects of stimulus probability on choice reaction time. Journal of Experimental Psychology, 92, 419-427.

Lappin, J. D., \& Disch, K. (1973). Latency operating characteristic: III. Temporal uncertainty effects. Journal of Experimental Psychology, 98, 279-285.

Marcel, A. J. (1983). Conscious and unconscious perception: Experiments on visual masking and word recognition. Cognitive Psychology, 15, 197-237.

Merikle, P. M., \& Reingold, E. M. (1998). On demonstrating unconscious perception: Comment on Draine and Greenwald (1998). Journal of Experimental Psychology: General, 127, 304-310.

Miller, J. (2000). Measurement error in subliminal perception experiments: Simulation analyses of two regression methods. Journal of Experimental Psychology: Human Perception \& Performance, 26, 1461-1477.

Naccache, L., Blandin, E., \& Dehaene, S. (2002). Unconscious masked priming depends on temporal attention. Psychological Science, 13, 416424.

Naccache, L., \& Dehaene, S. (2001). Unconscious semantic priming extends to novel unseen stimuli. Cognition, 80, 215-229.

Pinel, P., Rivière, D., Le Bihan, D., \& Dehaene, S. (2001). Modulation of parietal activation by semantic distance in a number comparison task. Neuroimage, 14, 1013-1026.

Smith, M. C., Besner, D., \& Miyoshi, H. (1994). New limits to automaticity: Context modulates semantic priming. Journal of Experimental Psychology: Learning, Memory, and Cognition, 20, 104-115.

Received December 27, 2001 Revision received August 2, 2002 Accepted August 12, 2002

\section{Members of Underrepresented Groups: Reviewers for Journal Manuscripts Wanted}

If you are interested in reviewing manuscripts for APA journals, the APA Publications and Communications Board would like to invite your participation. Manuscript reviewers are vital to the publications process. As a reviewer, you would gain valuable experience in publishing. The $\mathrm{P} \& \mathrm{C}$ Board is particularly interested in encouraging members of underrepresented groups to participate more in this process.

If you are interested in reviewing manuscripts, please write to Demarie Jackson at the address below. Please note the following important points:

- To be selected as a reviewer, you must have published articles in peer-reviewed journals. The experience of publishing provides a reviewer with the basis for preparing a thorough, objective review.

- To be selected, it is critical to be a regular reader of the five to six empirical journals that are most central to the area or journal for which you would like to review. Current knowledge of recently published research provides a reviewer with the knowledge base to evaluate a new submission within the context of existing research.

- To select the appropriate reviewers for each manuscript, the editor needs detailed information. Please include with your letter your vita. In your letter, please identify which APA journal(s) you are interested in, and describe your area of expertise. Be as specific as possible. For example, "social psychology" is not sufficient-you would need to specify "social cognition" or "attitude change" as well.

- Reviewing a manuscript takes time (1-4 hours per manuscript reviewed). If you are selected to review a manuscript, be prepared to invest the necessary time to evaluate the manuscript thoroughly.

Write to Demarie Jackson, Journals Office, American Psychological Association, 750 First Street, NE, Washington, DC 20002-4242. 